\title{
Gestión de la innovación para las Pymis de Ciudad Guayana
}

\author{
Salazar, Mauren * \\ Arzola, Minerva ** \\ Pérez, Elvira ***
}

\section{Resumen}

El presente artículo tiene como propósito diseñar un modelo para gestionar la innovación en las Pequeñas y Medianas Industrias, PYMIS, aplicado al caso de Ciudad Guayana, Estado Bolívar, Venezuela. El estudio se desarrolló en dos fases, en la primera fase se analizaron las bases teóricas existentes sobre gestión de la Investigación, Desarrollo e Innovación, I+D+I, explicando sus características en el ámbito nacional y en el ámbito mundial además del diagnóstico realizado a la actividad innovadora de veintitrés PYMIS; en la segunda fase se diseño y validó el modelo. Según los resultados, la PYMIS de Ciudad Guayana innovan en sus productos, con sus propios recursos, desconocen los programas públicos de apoyo a la innovación y el $75 \%$ de ellas invierten hasta un $5 \%$ de sus ventas en I+D+I. El modelo de innovación propuesto es abierto y completo; simple y aceptable; robusto y sensible; auto reflexivo, aplicable a toda la organización y portador de mensaje; contempla cinco aspectos de la gestión de la Innovación: Modelo de Procesos y Sistemas de Innovación, Responsabilidad de la Dirección, Gestión de los Recursos, Actividades de I+D+l y Medición, Análisis y Mejora.

Palabras clave: $\quad$ Modelo de Gestión, Innovación, PYMIS, I+D+I, Ciudad Guayana.

* Ingeniera Industrial. Magíster en Ing. Industrial. Consultor Independiente. Email: maurensc@ yahoo.com

** Dra. Ingeniero Industrial. Prof. Asociado-Investigadora. UNEXPO-Vicerrectorado Puerto Ordaz. Centro para el Desarrollo Gerencial. E-mail: minervarzola@yahoo.com

*** Ingeniero de Materiales. Magíster en Ingenieria Industrial. Profesora Invitada Maestria en Ingenieria Industrial. UNEXPO-Vicerrectorado Puerto Ordaz. E-mail: elviraperez@cantv.net 


\title{
Innovation Management for SMIs in Ciudad Guayana
}

\begin{abstract}
The objective of this project is to design a model to manage the innovation process in the small and medium industries, SMls. This model will be applied to Ciudad Guayana, in the State of Bolivar, Venezuela. The study was developed in two phases. First, the existing theoretical bases about managing research, development and innovation, R\&D\&l, were analyzed, explaining their characteristics in the Venezuelan and world arenas and a diagnosis of innovative activities in 23 SMls was performed. During the second phase, the model was designed and validated. According to the results, SMls in Ciudad Guayana innovate in their products, with their own resources. They do not know about public programs that support innovation, and $75 \%$ of them invest about $5 \%$ of their sales in R\&D\&I. The proposed model for innovation is open and complete; simple and acceptable; robust and sensitive; self-reflective, applicable to the entire organization and carries a message. This model considers five aspects of innovation management: a Model for Innovation Processes and Systems, Management Responsibilities, Resource Administration, R\&D\&I Activities and Measurement, Analysis and Improvement.
\end{abstract}

Key words: Management model, innovation, SMls, R\&D\&l, Ciudad Guayana.

\section{Introducción}

El fortalecimiento económico, la calidad de vida y la competitividad de un país dentro del contexto mundial actual, cada vez se encuentra mas relacionada a la gestión de la innovación. Por lo que ésta, debe ser el componente fundamental de toda política científica y tecnológica a nivel empresarial y nacional, además de ser un elemento clave que determina la posición de los países y de sus empresas dentro del mercado velozmente cambiante, competitivo y globalizado.

A nivel empresarial, la gestión de la innovación puede parecer muy compleja, esto se debe a que suele encontrarse con trabas técnicas que elevan sus costos. Muchas veces los procesos de I+D+I no se encuentran definidos, debido a que son procesos únicos, creativos y carentes de estructura. De igual manera son muchas las empresas que no la consideran al momento de elaborar los presu- puestos de gastos. A esto se le suma la falta de una infraestructura adecuada que permita el desarrollo de estas actividades y del personal con el tiempo y el conocimiento mínimo requerido para conformar la unidad de I+D+l. Además, de la ausencia de directrices para organizar y gestionar la Innovación.

En Latinoamérica las PYMIS tienden a la adquisición de tecnología y las mejoras continua, y prestan menor atención a la función de I+D+I; los esfuerzos para la realizar I+D+I no son registrados y son considerados implícitos en los procesos rutinarios de la organización.

En Venezuela la situación de las PYMIS es similar a la descrita, con la atenuante de la actual realidad económica, Genatio (2002). Tomando esta realidad como indicador de estudio, se puede decir que el balance productivo y económico de muchas de estas PYMIS es complejo y difícil. 
Gestión de la innovación para las Pymis de Ciudad Guayana

Salazar, Mauren; Arzola, Minerva y Pérez, Elvira

En Ciudad Guayana las PyMIS carecen de un modelo de gestión de innovación, que les permita canalizar los esfuerzos de innovar, definir, sistematizar, desarrollar y evaluar los procesos de $1+D+1$, Arzola (2001), Piñero et al (2007) y Rosales (1999). Tomando en cuenta que tienen como limitantes el tamaño, la capacidad de producción y recursos limitados. Esta situación provoca la fuga de ideas, productos y servicios innovadores, la desvalorización de la propiedad intelectual, el incremento del desempleo, el aumento de la capacidad ociosa de las empresas, la desactualización de la tecnología, y bajar la competitividad entre las PYMIS de la región y los mercados globales, Hurtado (2007).

La gestión de la innovación no solo incluye el hecho de presentar productos y servicios innovadores, si no, el de crear una estructura de gestión I+D+I, que pueda soportar todo la compleja naturaleza que esta comprende y las interacciones del mismo, por lo que se precisa de un modelo de gestión que permita sistematizar, evaluar y optimizar estos procesos.

Es importante resaltar que la realización del estudio proporciona información relevante, que sirve de base para el desarrollo competitivo de las PYMIS de Ciudad Guayana, desde el punto de vista de la innovación. Los resultados del estudio propuesto constituyen un aporte para el fortalecimiento del tejido industrial de la región; y representan un modelo que complementa el Modelo de Excelencia de Gestión de Ceproguayana, Mora (2004); ambos herramientas complementarias para mejorar la competitividad de la PYMIS de Ciudad Guayana.
El objetivo de este proyecto es diseñar un modelo para gestionar la innovación en las Pequeñas y Medianas Industrias, PYMIS, aplicado al caso de Ciudad Guayana, Estado Bolívar, Venezuela. La muestra analizada fueron veintitrés (23) PYMIS legalmente registradas, ubicadas en Ciudad Guayana adscritas a la Asociación de Industriales Metalúrgicos y de Minería de Venezuela, AIMM, Capítulo Regional Guayana; Cámara de Industriales y Mineros de Guayana, CIMG; y la Cámara de Comercio del Municipio Caroní, CAMCARONI.

La investigación se desarrollo en dos fases: a) una primera fase de tipo evaluativa, que permitió estimar de manera cualitativa la posición que tienen las PYMIS de Ciudad Guayana con respecto a la Innovación, utilizando una encuestas y la técnica del Focusgroup; y b) una segunda fase del tipo aplicada, el cual toma en cuenta los resultados de la primera fase evaluativa, para diseñar un Modelo de Gestión para la Innovación que permitirá a las PYMIS la organización y sistematización de sus procesos, proyectos y recursos de I+D+I, impulsando las actividades de innovación y garantizando la sobrevivencia en el mercado.

\section{Gestión de la innovación}

Según Betz (1997) la complejidad en la gestión de la innovación en las empresas viene dada por los diversos sistemas que constantemente están interactuando en el proceso de innovación, lo que da origen a un cambio permanente. Deben combinarse algunas técnicas para una gestión exitosa del proceso de innovación, tales como análisis organizacio- 
nal y de sistemas, planificación y predicción de la tecnología, gerencia de proyectos, marketing y técnicas para emprender nuevos negocios.

La gestión de la innovación requiere la comprensión de cómo ocurre este proceso en la empresas y organizaciones, los primeros investigadores de este tema planteaban que el proceso innovador ocurría de forma lineal y consecutiva, hasta que Klines (1985), propone la no linealidad del proceso innovador, describiéndolo como concurrente, cíclico, con cinco fases principales donde interactúan todas las unidades organizativas implicadas, con un objetivo común, acortar el tiempo de lanzamiento de los productos al mercado, ver Figura 1.

Esta herramienta permite conocer cada etapa de la innovación, así como también los parámetros y actores involucrados en todas sus fases. En este modelo se concibe la innovación como un proceso que comprende desde la generación de una idea hasta su explotación comercial. Involucra por lo tanto aspectos tangibles e intangibles muy complejos de dirigir; razón por la cual son muchas las investigaciones realizadas sobre la gestión eficaz del proceso de innovación. Importante destacar el aspecto de la retroalimentación, que sus fases ocurren concurrentemente e intervienen diferentes áreas funcionales tales como mercadeo, ingeniería, I+D, producción, comercialización, entre otras. Este modelo también prevé que el proceso de $1+\mathrm{D}+\mathrm{I}$ es cambiante e imprevisible, además de susceptible de sistematizarse y organizarse mediante un sistema de Gestión basado en la metodología conocida como. "Planificar-Hacer-Verificar-Actuar (PHVA) donde:

Figura 1.

Modelo cadena/eslabón del proceso de innovación tecnológica

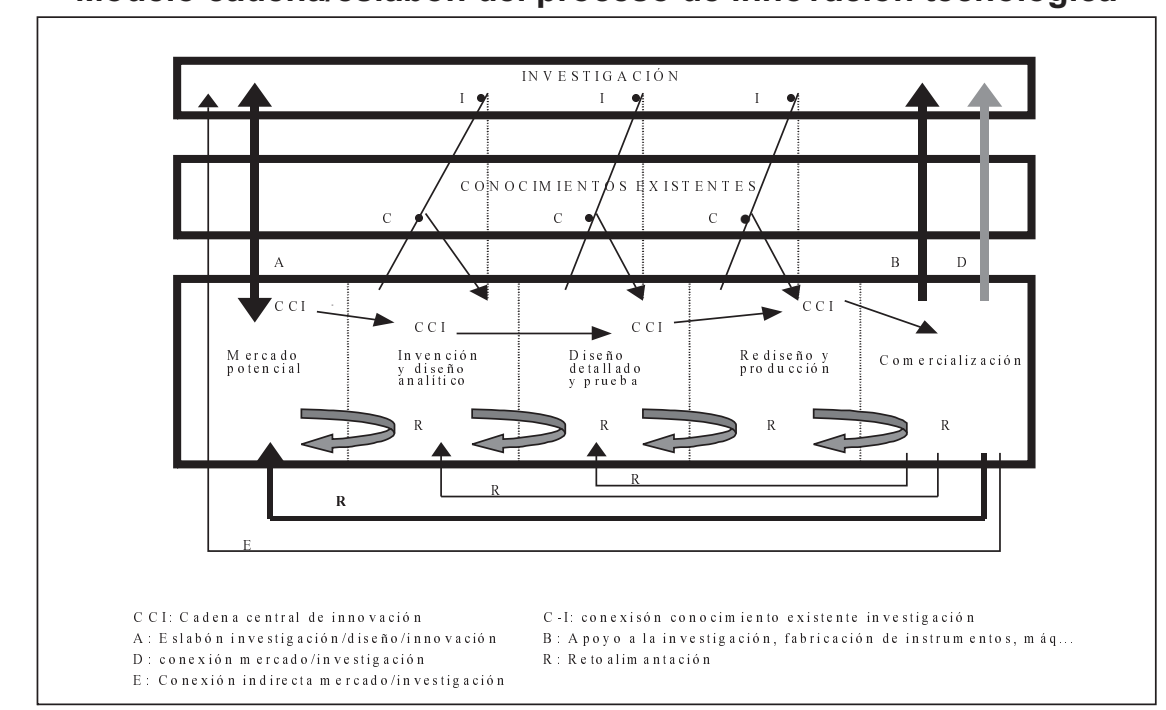

Fuente: Klines (1985). 
Gestión de la innovación para las Pymis de Ciudad Guayana

Salazar, Mauren; Arzola, Minerva y Pérez, Elvira

- Planificar: establece los objetivos necesarios de I+D+I para conseguir los resultados.

- Hacer: implantar el procedimiento de sistematización de la I+D+I.

- Verificar: Realizar el seguimiento y controlar el proceso de I+D+I respecto a los objetivos de $I+D+I$ e informar sobre los resultados.

- Actuar: Tomar decisiones para mejorar continuamente el proceso de I+D+l dentro de la Organización.

Según Maidique y Hayes (1997), no se pueden establecer relaciones causa-efecto duradera y estable en las empresas, pues el factor tecnológico se caracteriza por su gran dinamismo y mutabilidad. Pero también es cierto que la innovación, cuando es operativa, casi nunca es espontánea, y por lo tanto es importante su planificación y la incorporación de la dimensión tecnológica a la estrategia general del negocio.

Pavón e Hidalgo (1997), definen la gestión de la innovación tecnológica como "el proceso orientado a organizar y dirigir los recursos disponibles, tanto humanos como técnicos y económicos, con el objetivo de aumentar la creación de nuevos conocimientos, generar ideas que permitan obtener nuevos productos, procesos y servicios o mejorar los existentes, y transferir esas mismas ideas a las fases de fabricación y comercialización".

Morin (1985), plantea que una gestión eficaz del proceso innovador debería prestar especial atención a las funciones básicas, inventariar, vigilar, proteger, evaluar, enriquecer y optimizar. Markides (1997) es partidario que las empresas incorporen la cultura de innovación en su funcionamiento cotidiano y propone una rutina para iniciar este proceso de incorporación:

a) Redefinir el negocio.

b) Redefinir el quién: ¿quiénes son nuestros clientes?, pensar en nuevos clientes o nuevos segmentos y desarrollar planes para atenderlos mejor.

c) Redefinir el qué: ¿qué productos o servicios ofrecemos a esos clientes?, pensar en las nuevas necesidades o deseos de los clientes y desarrollar un plan que los satisfaga mejor.

d) Redefinir el cómo: aprovechar las competencias claves para desarrollar nuevos productos y mejorar el negocio para luego encontrar los clientes adecuados.

e) Iniciar el proceso mental en diferentes momentos en el tiempo: ¿cuáles son las capacidades singulares?, ¿qué necesidades específicas podemos satisfacer?, ¿quiénes serán los clientes adecuados que se deben atender?

La norma 166002 de la Asociación Española de Normalización y Certificación, AENOR (2004), denominada Requisitos del Sistema de Gestión de la I+D+I, se refiere a la gestión de la innovación y cuyo esquema de desarrollo es el de las normas ISO 9000:2000. En dicha normas destacan una descripción detallada de todas las actividades de $\mathrm{I}+\mathrm{D}+\mathrm{I}$ que las empresas deben cumplir para garantizar que se gestione adecuadamente la innovación, a saber: las herramientas para la I+D+I, identificación y análisis de problemas y oportunidades, análisis y selección de ideas de I+D+I, planificación, seguimiento y control de la cartera de proyectos, transferencia de tecnología, producto de la I+D+I, compras, resulta- 
dos del proceso de I+D+I y protección y explotación de los resultados de las actividades de $\mathrm{I}+\mathrm{D}+\mathrm{I}$.

En el Manual de Oslo (1997) se describen desde las definiciones de las actividades científicas, la medición del inputs y outpus tecnológicos, las clasificaciones institucionales y funcionales, y los métodos para elaborar trabajos estadísticos hasta los deflactores y tipos de cambio recomendados para igualar las mediciones de las diferentes variables en el tiempo.

La innovación científica y tecnológica, comprende todas las etapas científicas, técnicas, financieras y comerciales necesarias para el desarrollo y comercialización de productos manufacturados nuevos o mejorados, para la utilización comercial de nuevos o mejorados procesos y equipos, o para la introducción de un nuevo método de servicio social, un concepto más amplio que las actividades de $I+D$, las cuales constituyen sólo una de las etapas del proceso de innovación científica y tecnológica.

El Manual de Oslo agrupa los indicadores de ciencia y tecnología en tres categorías:

a) Indicadores de Recursos o de Inputs: considera dos tipos de inputs, los gastos en I+D y el personal empleado en estos trabajos durante cada año; que constituyen los recursos más significativos para desarrollar las actividades de I+D. En la cuantificación de los gastos de $1+D$, se plantea el problema de las diferencias de precios en los diferentes países. Para solventar esta situación, el manual también desarrolla los métodos de cálculo de deflactores especiales. b) Indicadores de Resultados o de Outputs: las estadísticas de patentes, la balanza de pago tecnológica, y los datos bibliométricos. Estos indicadores miden el resultado directo de la actividad científica y tecnológica, en forma de nuevos conocimientos y su impacto económico y social. Presentan dificultad en su uso, dada la naturaleza de las fuentes de información que los originan y la complejidad para su normalización.

c) Indicadores de Impacto: en este grupo se evalúan las transacciones comerciales de productos intensivos en tecnología y los índices de productividad. Estos indicadores representan el impacto de la ciencia y tecnología en la economía.

Esta metodología es una de la más difundida a nivel mundial, en todos los países, independientemente que pertenezcan o no a la OCDE y las estadísticas generadas son los más usados y discutidos en investigaciones sobres como medir la innovación tecnológica en los países y en las empresas.

El Manual de Bogotá (2002) es la versión para los países Latinoamericanos del Manual de Oslo, donde se realizan algunos ajustes y adaptaciones a las variables a evaluar dado el nivel de desarrollo tecnológico en estos países.

El modelo de aprendizaje tecnológico de Viana y Cervilla (1998), considera la evaluación de la innovación tecnológica como el resultado de un conjunto de variables estratégicas, de rendimiento o gestión, de capacidades técnico/gerenciales y de innovación interactuando en la empresa y con su entorno. El modelo hace énfasis en el desarrollo de capaci- 
Gestión de la innovación para las Pymis de Ciudad Guayana

Salazar, Mauren; Arzola, Minerva y Pérez, Elvira

dades tecnológicas en la empresa a través del aprendizaje tecnológico, lo que le permite adquirir habilidades y destrezas para instrumentar cambios en sus productos/servicios, procesos y sistemas. El aprendizaje tecnológico se refiere a todas aquellas actividades que realiza la empresa para construir y suplementar su base de conocimientos de sus tecnologías, productos y procesos; y para desarrollar y mejorar las destrezas de sus recursos humanos, a objeto de responder adecuadamente a las necesidades del mercado.

El proceso de desarrollo de capacidades tecnológicas tiene tres aspectos principales, la capacidad para adquirir tecnología, la capacidad de uso de la tecnología y la realización de actividades de $I+D$, de ingeniería y de diseño.

A partir de 1998, la Unión Europea para poder lograr sus estrategias de crecimiento económico y competitividad se plantea la necesidad de contar con una herramienta de medición de la innovación en sus países miembros que a su vez pueda ser utilizada también por las empresas. Desarrollan entonces una metodología denominada Cuadro Europeo de Indicadores de Innovación, Innovación y Transferencia Tecnológica (1999), y desde el año 2001 comenzaron a generar estadísticas de sus actividades Científicas, Tecnológicas y de Innovación. El Cuadro Europeo de Indicadores de Innovación evalúa cuatro grandes áreas, a saber: Generación de Conocimientos, Promoción de la Innovación, Actividades de Innovación y Financiamiento de la Innovación. Este proceso de medición, les ha permitido ajustar y adaptar sus políticas de promoción y apoyo a la I+D+I.
Kaplan y Norton (2004), proponen a la empresa el desarrollo de su mapa estratégico con el propósito de crecer sostenidamente y generar valor para sus accionistas. Este mapa estratégico se fundamenta en atender las cuatro perspectivas del Cuadro de Mando Integral: financiera, cliente, interna, de aprendizaje y crecimiento; cuyo despliegue estará en función al tipo de empresa y a la actividad económica a la que ésta pertenezca.

Este modelo de mapa estratégico además de proponer las cuatro perspectivas de funcionamiento de la empresa, propone que la creación de valor se origina a través de los procesos internos, donde la innovación bien gestionada garantiza el crecimiento y la competitividad del negocio. Adicionalmente, proporciona un lenguaje común para establecer prioridades de crecimiento para la empresa, así como también una lista normativa de los componentes e interrelaciones. Los indicadores estratégicos de resultados muestran una serie de vínculos causa-efecto entre los activos intangibles y los procesos de creación de valor en términos con los objetivos de las cuatro perspectivas del mapa.

\section{Situación Actual de la Gestión de la Innovación en las PYMIS de Ciudad Guayana}

Es importante reseñar que las empresas analizadas ofrecen productos $y$ servicios variados, las cuales pertenecen a los sectores comercio, metalmecánica, salud, electrónica, construcción, electricidad, y radiocomunicación. De esta primera experiencia, se puede observar que la alta gerencia de estas PYMIS tiene definido su visión del negocio; es decir, saben 
lo que quieren y hacia donde van, además cuentan con mucha experiencia y preparación.

La caracterización de la actividad innovadora de las PYMIS de Ciudad Guayana, se realizó mediante la evaluación de las principales variable que definen el proceso innovador, a saber: tipos y frecuencia, planificación, recursos asignados, uso de programas públicos, inversión en I+D+I, nivel académico del personal y el mejoramiento continuo.

En el Grafico 2, se observa que en los últimos 5 años, el $75 \%$ de las PYMIS de Ciudad Guayana innovaron en sus productos y servicios, el $50 \%$ en sus procesos, el $62,5 \%$ en aspectos organizativos y sólo el 37,5 innovo en tecnología. En cuanto si las PYMIS consultadas contemplan la Innovación dentro de sus planes y objetivos empresariales, el $100 \%$ de estas empresas afirman que dentro de sus planes y objetivos estratégicos se encuentra inmerso el desarrollo de la innovación de sus productos, servicios y procesos, pues se ven obligadas estar a la vanguardia por tipo de producto y servicio que ofrecen, debido a que su mercado $y$ cartera de clientes les exige cambios de manera constante.

En cuanto al origen de los recursos utilizados para lograr innovar, se aprecia que el $87,5 \%$ de las PYMIS de Ciudad Guayana innovan y mejoran el diseño de sus productos y procesos haciendo uso de sus propios recursos, el 50\% contrata los servicios de empresas especializadas, el $25 \%$ se apoya con Universidades o Institutos Educativos, y $25 \%$ realiza alianzas con empresas grandes o se apoyan con la planta que les comercializa su producto, ver Gráfico 3.
Gráfico 2

Tipos de Innovación de las PYMIS de Cd. Guayana

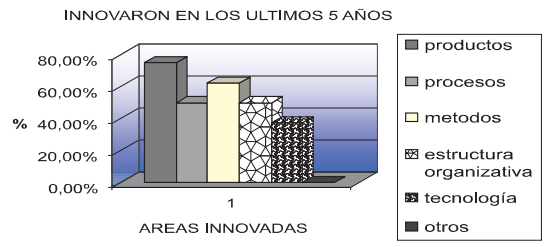

Gráfico 3

Origen de los recursos usados para innovar por las PYMIS de Ciudad Guayana

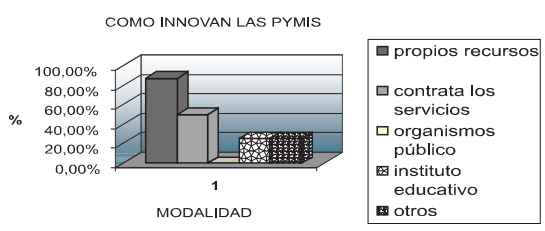

Según la muestra analizada, el $75 \%$ de las PYMIS de Ciudad Guayana conocen muy poco o desconocen los programas públicos que apoyan la actividad innovadora empresarial existentes en la región y el país, ver Gráfico 4.

En cuanto a la reducción de costos y aumento de ganancias, las PYMIS de Ciudad Guayana consultadas manifestaron su necesidad de reducir los costos y contar con herramientas que le permitan generar más ganancias a sus empresas.

De igual manera, afirmaron que la idea de contar con una unidad organizativa de $\mathrm{I}+\mathrm{D}+\mathrm{I}$ es buena siempre y cuando no aumente sus costos; por otra parte, como resultado de la encuesta aplicada es claro que el $75 \%$ de ellas invierten entre un $0.1 \%$ a $5 \%$ de sus ingresos para realizar trabajos de investigación y poder 


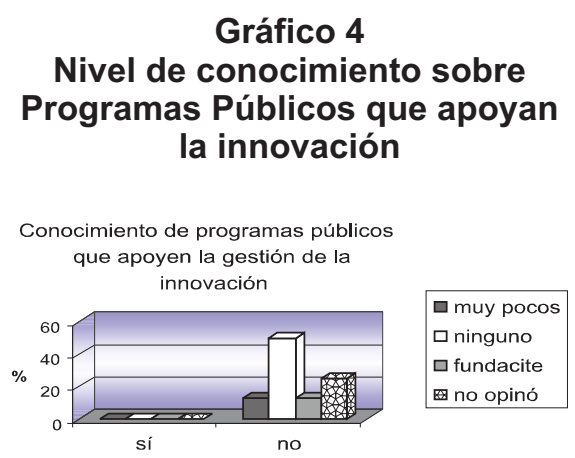

innovar en sus servicios, productos o mejorar sus métodos de trabajo, el otro $25 \%$ no realiza ningún tipo de inversión, ver Gráfico 5

El $50 \%$ de las empresas consultadas poseen una cultura de mejoramiento continuo el $50 \%$ restante no opino. En cuanto al nivel académico del personal que labora en las PYMIS, el $100 \%$ manifiestan contar con personal que posee preparación universitaria y representan entre un $10 \%$ hasta el $65 \%$ del total de empleados que laboran en sus empresas.

\section{Estrategias para mejorar la gestión de la innovación en las PYMIS de Ciudad Guayana}

Complementando y combinando los resultados de la encuesta aplicada, la información obtenida en la dinámica del Focusgroup con los empresarios y el análisis del entorno, se identificaron las Fortalezas, Debilidades, Oportunidades y Amenazas en la gestión de la innovación en la PYMIS de Ciudad Guayana.

\section{OPORTUNIDADES}

- Los programas de apoyo al proveedor de productos y servicios que lle-

\section{Gráfico 5 \\ Inversión en I+D+I en las PYMIS de Ciudad Guayana}

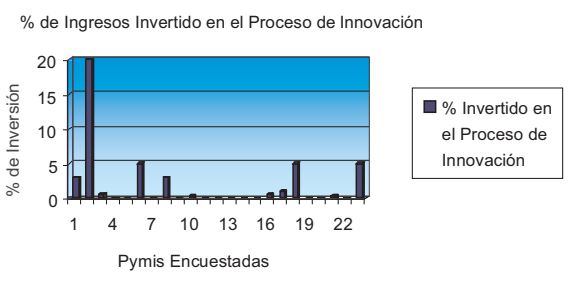

van a cabo grandes empresas, entre ellas Ternium SIDOR.

- Los programas de apoyo para el desarrollo de productos innovadores que lleva cabo el Gobierno Nacional.

- La creación de productos innovadores diseñados por Investigadores que se apoyan en esquemas del Gobierno.

- La creación de parques industriales en otros estados del país, con miras a desarrollar productos Innovadores.

- La existencia de Universidades e Institutos Educativos que llevan a cabo el desarrollo de productos innovadores.

- Ley de Ciencia, Tecnología e Innovación, LOCTI.

\section{AMENAZAS}

- El control cambiario y la falta de políticas claras.

- Escasa divulgación de las políticas de innovación por parte del Estado.

- La aparición de empresas fantasmas con productos novedosos.

- La incertidumbre ante la situación actual del país.

- La falta de instituciones que brinden adiestramiento especializado.

- La calidad se compara en base al precio de los productos y servicios. 


\section{FORTALEZAS}

- La relación directa de las PYMIS con sus clientes.

- La experiencia y preparación de la alta gerencia.

- Contar con profesionales universitario que puedan desarrollar los procesos de Innovación.

- Existe compromiso y dedicación por parte de la alta gerencia.

- Ubicación geográfica favorece la obtención de materias primas.

- La cultura de mejoramiento continuo en sus productos, procesos y servicios.

- Planes y objetivos estratégicos donde se encuentra inmerso el proceso de innovar.

- Contar con personal con experiencia.

\section{DEBILIDADES}

- Elevados costos de operaciones.

- Falta de conocimiento de programas públicos y otros que puedan apoyar el desarrollo de productos y servicios Innovadores.

- Poco uso de herramientas técnicas para la gestión general y de la actividad innovadora.

- No manejar de manera amplia el concepto de innovar y todo lo que ello implica puede convertirse en una debilidad.

- A pesar de contar con el personal preparado y con experiencia, si no se motiva a la ejecución de los proyectos de innovación puede convertirse en una debilidad.

- El incremento de la morosidad de la cobranza puede afectar que los recursos no se destinen a la investigación y a gestionar la innovación.
- No contar con una unidad que desarrolle investigación y gestione la innovación en estas empresas.

A partir de análisis FODA de la actividad innovadora en la PYMIS de Ciudad Guayana, se generaron un conjunto de acciones estratégicas parara mejorar esta área en las empresas, ver Cuadro 1.

\section{Características del modelo de gestión de la innovación para las PYMIS}

Una vez realizado el diagnóstico de la situación actual en cuanto a la posición de las PYMIS Ciudad Guayana, se propone el Modelo de Gestión de la Innovación, el mismo trata de conservar y complementar el diseño de los modelos basados en los Modelos de Excelencia Organizacional existentes, y sus características son:

1. Abierto y completo. El modelo debe incluir todas las áreas de la empresa, cubrir todos los niveles jerárquicos y poder ser aplicable en las distintas fases de madurez en el ciclo de vida de la empresa.

2. Flexible. A pesar de los cambios que se puedan suscitar debido al mismo proceso de innovación este, debe adaptarse con facilidad a los mismos incluyendo el entorno competitivo, los cambios de políticas y estrategias y de los cambios de estilo de dirección.

3. Simple/Aceptable. El modelo deberá ser "fácil de entender" y "fácil de aplicar". Debe ser comprendido y utilizado por personas de la organización aun cuando no estén familiarizados con modelos organizacionales y de Innovación. 
Gestión de la innovación para las Pymis de Ciudad Guayana

Salazar, Mauren; Arzola, Minerva y Pérez, Elvira

\section{Cuadro 1}

Estrategias para mejorar la innovación en las PYMIS de Ciudad Guayana.

\begin{tabular}{|c|c|c|}
\hline & OPORTUNIDADES & AMENAZAS \\
\hline & "Estrategias ofensivas" & "Estrategias defensivas" \\
\hline \multirow[t]{2}{*}{ Fortalezas } & $\begin{array}{l}\text { Establecer convenios e intercam- } \\
\text { bios de experiencias y conocimien- } \\
\text { tos con las empresas básicas que } \\
\text { brinden programas de apoyo al pro- } \\
\text { veedor, con el fin de fortalecer al } \\
\text { personal profesional y de experien- } \\
\text { cia y esquematizar una nueva forma } \\
\text { de hacer las cosas. } \\
\text { De igual forma, lucrarse de los pro- } \\
\text { gramas de innovación auspiciados } \\
\text { por el gobierno. }\end{array}$ & $\begin{array}{l}\text { Establecer una vigilancia continua } \\
\text { en cuanto a los cambios que puedan } \\
\text { generarse en las políticas del go- } \\
\text { bierno y flexibilizar las estrategias } \\
\text { utilizadas con el fin de que puedan } \\
\text { ser adaptadas a los nuevos cambios } \\
\text { que puedan suscitarse }\end{array}$ \\
\hline & "Estrategias de reorientación" & "Estrategias de supervivencia" \\
\hline Debilidades & $\begin{array}{l}\text { Definir un esquema de orientación, } \\
\text { o guía metodológica a seguir para } \\
\text { gestionar la innovación, este debe } \\
\text { incluir el aprovechamiento de las } \\
\text { oportunidades que agentes exter- } \\
\text { nos puedan ofrecer. }\end{array}$ & $\begin{array}{l}\text { Motivar al personal y crear una cul- } \\
\text { tura de desarrollo de ideas y proyec- } \\
\text { tos innovadores que permitan no } \\
\text { solo aumentar la rentabilidad de la } \\
\text { empresa si no disminuir los costos } \\
\text { en ella. }\end{array}$ \\
\hline
\end{tabular}

Fuente: Elaboración propia.

4. Robusto/Sensible. El modelo debe soportar el sistema de dirección el cual no se cambia cada año. Incluso si algún factor del entorno cambia, el modelo no debería cambiar.

5. Auto reflexivo/Innovador. El modelo deberá animar a la innovación desde la reflexión en un marco actual realista y consolidado, tomando en cuenta las áreas que puedan estar afectadas por las actividades de I+D+I.

6. Aplicable a la organización como un todo y a cada unidad de la empresa. El modelo debe ser aplicable a la organización como un todo y al mismo tiempo, a cada unidad de negocio, incluso a cada área funcional.

7. Portador de mensajes. El uso del Modelo no debe acabar en sí mismo, sino que debe llevar mensajes añadidos, como el de la búsqueda de la mejora continua y la competitividad.

\section{Modelo de Gestión de la Innovación para las Pequeñas y Medianas Industrias, PYMIS}

El modelo de Gestión de la Innovación para las PYMIS de Ciudad Guayana toma en cuenta los criterios propuestos en los modelos anteriores y adapta los aspectos planteado por la Norma Española Experimental 166000 de la Gestión de la I+D+I, el Cuadro Europeo de Indicadores, Manual de Oslo y Manual de Bogota y que pueden ser aplicados dado las características de las PYMIS de la Ciudad Guayana. 
El Modelo de Gestión de la Innovación, ver Diagrama 1, evalúa cinco (05) aspectos del Sistema de la Gestión de I+D+l complementando así, el Modelo de Excelencia de Gestión, a continuación se nombra los aspectos:

- Modelos de procesos y sistema de la $\mathrm{I}+\mathrm{D}+\mathrm{I}$.

- Responsabilidad de la Dirección.

- Gestión de los recursos.

- Información y análisis.

- Actividades de I+D+I.

- Medición, Análisis y Mejora.

1. INNOVACIÓN: Según el Manual de FRASCATI (OCDE, 1980). Es la transformación de una idea en un producto vendible nuevo o mejorado, o en un proceso operativo en la industria y en el comercio, o un nuevo método de servicio social. Esta puede ser clasificada en dos rubros, la llamada Innovación de Producto el cual se basa en la Fabricación y comercialización de nuevos productos o mejores versiones de productos existen- tes y la Innovación por Proceso enfocada hacia la Instalación de nuevos procesos de producción para mejorar la productividad o racionalizar la fabricación, ya sea para la fabricación de productos nuevos o para la fabricación más eficiente de productos existentes.

\section{MODELOS DE PROCESOS $Y$}

SISTEMAS DE I+D+l: Los procesos así como los sistemas de I+D+I deben ser establecidos, documentados e implantados, para esto, la organización debe identificar las actividades propias de I+D+I, determinar su secuencia e interacción, al igual que los criterios y métodos necesarios para asegurar un eficaz funcionamiento y control.

Un modelo de Proceso de $1+\mathrm{D}+1$ puede ser fácilmente representado a través del modelo modificado de "enlaces de cadena" de Kline, el cual refleja la complejidad e incertidumbre que implica un proceso de $\mathrm{I}+\mathrm{D}+\mathrm{I}$ y la relación entre la innovación y las actividades de investigación y desarrollo.

\section{Diagrama 1 \\ Modelo para la gestión de la innovación en las PYMIS de Ciudad Guayana}

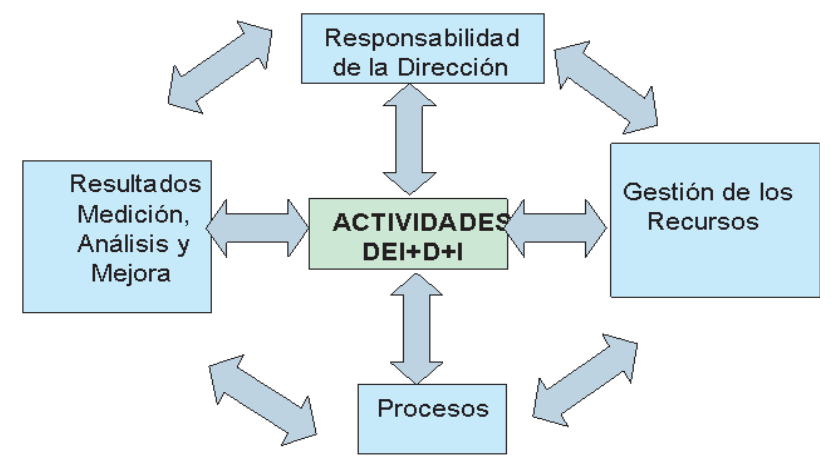

Fuente: Elaboración propia. 
Gestión de la innovación para las Pymis de Ciudad Guayana

Salazar, Mauren; Arzola, Minerva y Pérez, Elvira

El proceso de $\mathrm{I}+\mathrm{D}+\mathrm{I}$ es cambiante $\mathrm{e}$ imprevisible, pero puede ser sistematizado y organizado mediante un sistema de gestión de I+D+I, tal como lo plantea la norma española experimental "Gestión de la I+D+I", este, se basa en la utilización de la metodología PHVA (Planificar-Hacer-Verificar-Actuar) donde:

- Planificar: considera el establecimiento de los objetivos de I+D+I necesarios para conseguir los resultados de $\mathrm{I}+\mathrm{D}+\mathrm{I}$ de acuerdo con la estrategia tecnológica marcada por la dirección y los requisitos del mercado potencial.

- Hacer: implanta el procedimiento de sistematización de I+D+I.

- Verificar: Realiza el seguimiento y controla el proceso de I+D+I respecto a los objetivos de I+D+l e informar sobre los resultados.

- Actuar: Se toman decisiones para mejorar continuamente el proceso de I+D+I dentro de la organización.

3. RESPONSABILIDAD DE LA DI-

RECCIÓN: La alta dirección debe estar seriamente comprometida con el desarrollo e implantación del sistema de gestión de I+D+I, por lo que debe asegurarse de comunicar a la organización la importancia de la misma y participar de manera activa en el desarrollo de políticas, objetivos, planes, así como de asegurar la disponibilidad de los recursos y la creación de la unidad gestora de I+D+I.

Dentro de este aspecto, la alta dirección debe asegurar también, de que las funciones y responsabilidades de una unidad de gestión de I+D+I estén definidas y acordes con el propósito de la organización.

\section{GESTIÓN DE LOS RECUR-}

Sos: evalúa la utilización y administración de los recursos en la etapa de implantación y de mantenimiento de la unidad de gestión de I+D+I, de igual manera, examina las estrategias de la Organización para seleccionar el recurso humano y desarrollar su potencial a través de la motivación, competencia, toma de conciencia y formación, así como también, propiciar el alto desempeño y, participación. Considerando que se trata de PYMIS, las mismas en su mayoría no poseen una infraestructura física adecuada para el desarrollo de las actividades de $I+D+I$, por lo que se evaluará como Unidad de Gestión de I+D+l el recurso humano, material y físico que estén relacionados con esta actividad.

5. ACTIVIDADES DE I+D+l: Dentro de las actividades básicas de $|+D+|$ se debe considerar : el proceso de vigilancia tecnológica, la identificación de las necesidades de información tecnológica de la organización, análisis de problemas y oportunidades, la creatividad, la innovación tecnológica, la difusión de la información, análisis y selección de ideas, planificación, seguimiento y control de la cartera de proyectos, sistematización de la transferencia tecnológica, el estudio de mercado y la planificación y desarrollo del proceso necesario para desarrollar un producto de I+D+I.

6. MEDICIÓN ANÁLISIS Y MEJO-

RA: La organización debe planificar, programar e implantar los procesos de seguimiento, medición y control de los procesos y sus resultados, análisis de los datos, mejora continua y tomar las acciones correctivas y preventivas para eliminar las causas de no conformidades. 
Partiendo de la premisa de que muchas de las PYMIS que se encuentran en la Ciudad Guayana presentan procesos y productos innovadores, este modelo, permitirá la auto evaluación de las posibles áreas que no se han desarrollado hasta el momento, dando la oportunidad de que sean tomadas en cuenta para futuros lineamientos y estrategias de acción.

Cada uno de estos aspectos tiene una ponderación expresada en puntos, tal como se muestra a continuación en la Tabla 1.

\section{Conclusiones}

- La gestión de la innovación en las Pymis de Guayana se realiza empíricamente mas no sigue una metodología o procedimiento coherente y ordenado para detectar todos los elementos que implica Innovar.

- Las PYMIS de Ciudad Guayana innovan en sus productos, con sus propios recursos, desconocen los programas públicos de apoyo a la innovación y el $75 \%$ de ellas invierten hasta un $5 \%$ de sus ventas en I+D+I.
- Las empresas estudiadas innovan y mejoran el diseño de sus productos y procesos haciendo uso de sus propios recursos, contratando los servicios de empresas especializadas, se apoyan con Universidades o Institutos educativos, realizan alianzas con empresas grandes y se apoyan con la planta que les comercializa su producto, de aquí, se aclaro que no hacen uso de los esquemas o programas públicos existentes para impulsar la Innovación.

- Las características del modelo de Gestión de la Innovación propuesto se contempla de que sea abierto y completo; simple y aceptable; robusto y sensible; auto reflexivo e innovador; aplicable a la organización como a un todo y a cada unidad de la empresa y portador de mensaje.

- El modelo de innovación propuesto, pretende motivar el interés de las PYMIS en el desarrollo de actividades de Innovación, como elemento clave para asegurar la competitividad de la empresa y distinguirla del resto elevando su prestigio. Además de fomen-

Tabla 1

Ponderación de los aspectos a evaluar. Modelo de gestión de innovación para las PYMIS de Ciudad Guayana.

\begin{tabular}{lcc}
\multicolumn{1}{c}{ Aspectos } & $\%$ & Puntos \\
\hline 1. Modelos de Procesos y Sistemas de Innovación & 20 & 200 \\
2. Responsabilidad de la Dirección. & 20 & 200 \\
3. Gestión de los Recursos. & 20 & 200 \\
4. Actividades de I+D+I. & 25 & 250 \\
5. Medición Análisis y Mejora. & 15 & 150 \\
Total & 100 & 1000 \\
\hline
\end{tabular}

Fuente: Elaboración propia. 
Gestión de la innovación para las Pymis de Ciudad Guayana

Salazar, Mauren; Arzola, Minerva y Pérez, Elvira

tar las actividades de I+D+I, proveer de un Instrumento que permita la Autoevaluación en aquellas áreas que puedan tener Impacto de Innovación y brindar la herramienta básica necesaria para organizar y gestionar de manera eficaz la Innovación, aun, sin contar con una unidad física de $1+D+1$.

- El Modelo de Innovación propuesto contempla cinco aspectos de la gestión de la Innovación, dentro de los cuales podemos nombrar: Modelo de Procesos y Sistemas de Innovación, Responsabilidad de la Dirección, Gestión de los recursos, Actividades de I+D+I y Medición, Análisis y Mejora.

\section{Referencias Bibliográficas}

Asociación Española de Normalización y Certificación AENOR (2006). Norma 166002. Gestión de la I+D+i: Requisitos del Sistema de Gestión de la I+D. Madrid. 24p.

Arzola, Minerva (2001). Comparación de la Capacidad Tecnológica del Sector Metal entre Aragón/España y Guayana/Venezuela mediante Categorías Universales: Perspectivas para la Cooperación Empresarial. Tesis Doctoral. Universidad de Zaragoza. España. Pp. 1-27.

Betz, Frank (1998). Managing Technological Innovation, Competitive Advantage from Change. John Wiley \& Sons, Inc. New York.

Comisión Europea (1999). Cuadro Europeo de Innovación. Revista Innovación y Transferencia Tecnológica. Edición Especial. Dirección General de Empresas e Industria. Bruselas.

Genatios, Carlos; La Fuente, Marianela (2004). Ciencia y tecnología en Venezuela. Ediciones OPSU. Caracas.
Kaplan, Robert y Norton, David (2004). Mapas Estratégicos. Ediciones Gestión 2000. Barcelona. España.

Hurtado, Eliú (2007). Diseño de estrategias para el fortalecimiento del sector financiero en apoyo a las actividades de I+D+I de las PYMI en el área de materiales y metalmecánica del estado Bolívar. Trabajo de Grado Maestría Ingeniería Industrial. Unexpo. Vicerrectorado Puerto Ordaz.

Kline, Stephen (1985). Innovation is not linear process. Research Management. Julio-Agosto. Pp. 36-45.

Maidique, Roberto y Hayes Robert (1988). Strategic Management of Technology and Innovation. Richard D. Irwin, Inc.

Mora, Scandra (2004). Evaluación de la posición competitiva y diseño de un modelo de excelencia para la sobrevivencia y recuperación de las PyMIS de Ciudad Guayana ante mercados globalizados. Trabajo de Grado Maestría Ingeniería Industrial. Unexpo. Vicerrectorado Puerto Ordaz.

Morin, Jacques (1985). L'excellence technologique. Publi Union. Paris. 177p.

Organisation for Economic Coooperation and Development, OECD (1997). Oslo Manual. Bruselas.

Organisation for Economic Cooperation and Development, OECD (1980). Frascati Manual. Paris.

Pavón, Julian y Hidalgo, Antonio (1997). Gestión e Innovación, un Enfoque Estratégico. Ediciones Pirámide. Madrid. Pp. 63-84.

Piñero, Edgar; Arzola, Minerva (2007). Capacidades y potencialidades endógenas de centros, laboratorios de centros de investigación y desarrollo tecnológicos, en las áreas de materiales y mecánicas de Ciudad Guayana, Venezuela. Internacional Conference on Industrial Engineering and Industrial 
management, ClO2007. Universidad Politécnica de Madrid. September 5-7. Pp. 235-236. Book of Abstracts. ISBN: 978-84-611-8245-9.

Red Iberoamericana de Indicadores de Ciencia y Tecnología, RICYT (2001). Manual de Bogotá. Bogotá.

Rosales, Ramón (1998). Potencial competitivo de la PYMI del estado Bolívar; "Informe Final”. FONFI Bolívar (Venezuela).
Organización de Cooperación y Desarrollo Económica, OCDE (1992). Manual de Frascati. Paris.

Viana, Horacio y Cervilla, María (1998). Tecnología y competitividad en la industria manufacturera. Fondo Fintec. Caracas. 